\title{
Fabrication and packaging of integrated chemo-optical sensors
}

\author{
R.G. Heideman*, G.J. Veldhuis, E.W.H. Jager, P.V. Lambeck \\ MESA Research Institute, University of Twente, P.O. Box 217, 7500 AE Enschede, The Netherlands
}

\begin{abstract}
This paper describes the design and fabrication of a sensitive integrated chemo-optical sensor supplied with on-chip fiber-towaveguide connectors. The sensor is designed for TE-polarized light with wavelength of $633 \mathrm{~nm}$. The fiber-to-chip connectors are based on easily fabricated silicon V-grooves combined with a smooth sawcut. The sawcut is defining the channel waveguide endface. The sensor is based on a phase modulated Mach-Zehnder interferometer, using the electro-optic effect of the waveguiding material zinc oxide $(\mathrm{ZnO})$. The fiber-to-chip connector units have a typical coupling efficiency of $0.1-1 \%$. The electro-optical voltage $\times$ length product $V_{\pi}$ is $15 \pm 4 \mathrm{~V} \mathrm{~cm}$ at frequencies above $100 \mathrm{~Hz}$. Preliminary experiments on the general (passive) sensor response showing its expected high sensitivity are discussed.
\end{abstract}

Keywonds: Chemo-optical sensor; Zinc oxide (ZnO); Electro-optic; Phase modulation; Mach-Zehnder interferometer; Fiber-to-chip coupling

\section{Introduction}

There is a growing need for sensitive chemical sensors in several industrial areas such as process technology, health care, environmental control, biotechnology, etc. $[1,2]$. For commercial applications these sensors generally should be very sensitive, small, fast, and cheap, and have the possibility of remote sensing. An attractive solution fulfilling all these requirements can be formed by integrated optical (IO) sensors [3]. Here the chemical parameter to be detected influences the propagation properties of the light. Especially sensors using the evanescent field of an optical waveguide (see Fig. la) can be very sensitive, as the interaction length can be relatively long (several $\mathrm{mm})[3,4]$. With these sensors a chemically induced variation of the refractive index wit in the penetration depth of the evanescent field (typicaily several hundreds of nanometers) is transformed into a phase change. In the IO sensor field the Mach-Zehnder interferometer (MZI) sensor is generally supposed to have the highest potential [3,7]: the interferometer geometry is especially suited for detecting the analyte induced optical phase change, while the Mach-Zehnder configuration is intrinsically balanced

\footnotetext{
* Corresponding author.
}

due to the incorporation of a reference branch (see Fig. lb), resulting in a stable sensor signal:

$I_{\text {output }} \sim I_{\text {input }}(1+\cos (\Delta \varphi))$

where $\Delta \varphi$ is the phase change induced by the external parameter to be sensed.

As the selectivity of these integrated optical sensors is completely determined by the immobilized transducer layer, a large sensor family can result: immunosensors throuch antibady nroteins [4], ammonia sensors through bromocresol purple (BCP) [5], etc.

Despite its strong points, the integrated optical MZI has seldom been applied for commercial sensor applications until now. This is probably due to two major drawbacks. Firstly, there is the general problem of connecting the optical chip via fibers to external parts, i.e. the light source and the detection unit, in an economical way. Secondly, the MZI sensor signal is not easily linearized (see Eq. (1)), which is essential for exploiting the high sensitivity potentials $[6,7]$.

By using zinc oxide $(\mathrm{ZnO})$ optical waveguides on silicon substrates, both problems can be overcome. For such IO chips we have developed a simple self-aligning fiberto-chip coupling scheme, enabling quick and reliable 
connection of the fiber to the optical chip without loosing the high sensor sensitivity. This coupling scheme is essentially based on photolithographically defined $\mathrm{V}$ grooves in the silicon substrate, combined with a sawcut. The sawed $\mathrm{ZnO}$ optical waveguide endface shows small roughness only, so polishing is not needed. Furthermore, the shown thin film properties of $\mathrm{ZnO}$ as discussed in Ref. [6] allow for electro-optically induced phase modulation, which can be used for linearizing the sensor signal [7].

In this contribution we present the scheme we have developed for the fabrication of an optical chip, consisting of a phase modulated integrated Mach-Zehnder interferometer sensor, combined with self-aligning fiber-tochip connection units. Emphasis is laid on integration instead of optimization of the different components. The design and fabrication of the complete device are discussed: this includes the fiber-to-chip connection unit, the Mach-Zehnder interferometer sensor design and the waveguide configuration used.

With a prototype of the packaged devices, preliminary measurements were performed. The corresponding fiberto-chip coupling efficiency and the electro-optic phase modulation results are discussed. Furthermore, preliminary refractive index measurements were performed with these devices, showing the high general sensor sensitivity: specific selectivity measurements will be presented in the near future.

a

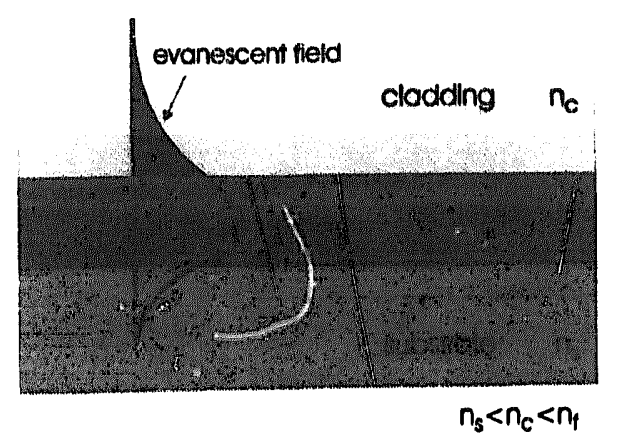

b

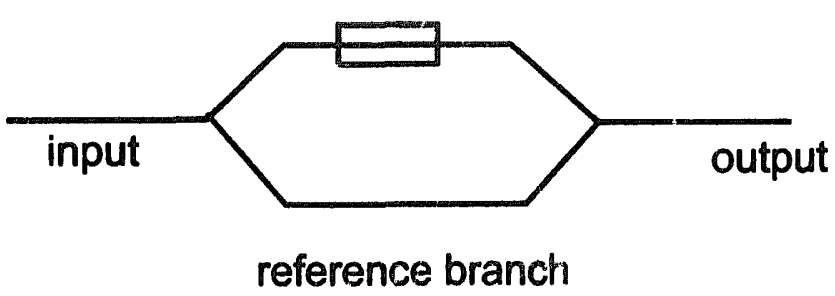

Fig. 1. Cross-section of an optical waveguide, showing the sensing modal evanescent field: the cladding material is accessible for the analyte to be detected (a). Top view (schematic) of a basic integrated Mach-Zehnder interferometer consisting of channel waveguides, with (part of) the sensor branch subject to the external parameter to be sensed (b).
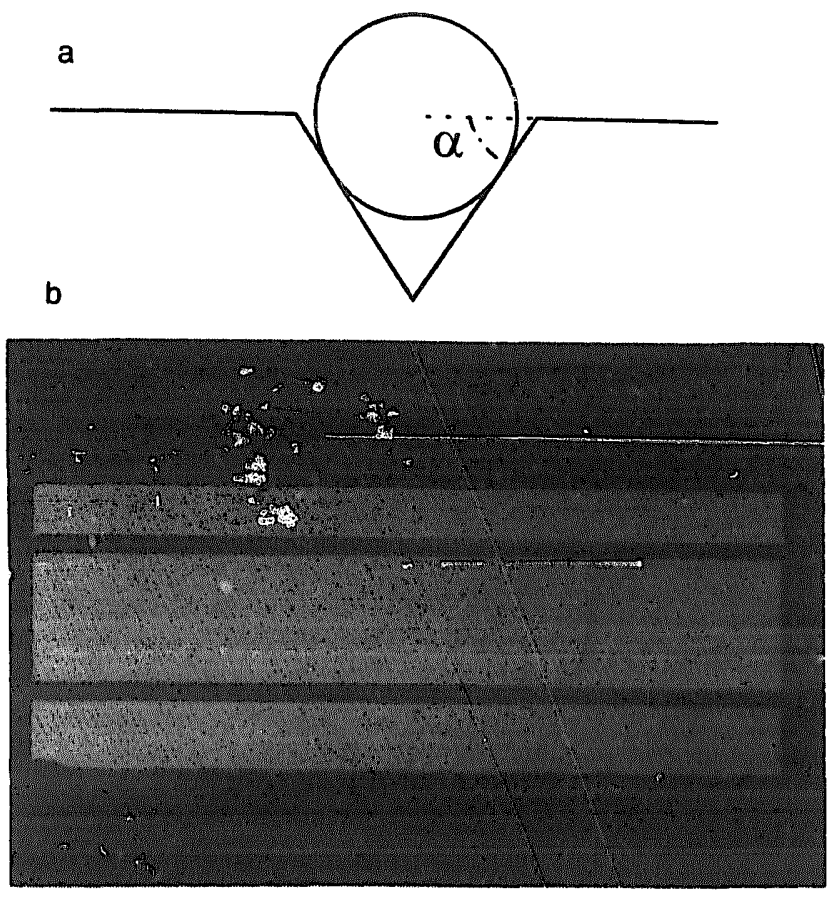

Fig. 2. Schematic representation (cross-section) of a fiber in a V-groove in silicon (a). Photograph (top view) of a Si-V-groove, showing the wet etched smooth crystal planes, ensures a perfectly controlled depth, width and orientution of the groove.

\section{Fiber-to-chip connection unit}

In contrast to telecommunication applications, most sensor applications do not require an optimized coupling efficiency between optical fiber and integrated optical channel waveguide. More important for achieving commercially attractive integrated optical sensors, is a low cost, reproducible connection of optical fibers to the channel waveguides on the chip [8-10]. Therefore a selfaligning fiber-to-chip connection unit was developed which requires no polishing steps and no external fiberto-chip alignment equipment.

The starting point of our fiber-to-chip connection unit is the well known wet ctched V-shaped groove in a silicon (Si) wafer using a $\mathrm{KOH}$ solution [11] (see Fig. 2). The V-groove results from the large difference in $(\mathrm{KOH})$ etch rate of the different Si-crystal planes: 100:16:1 for the $\langle 100\rangle,\langle 110\rangle$ and $\langle 111\rangle$ plane, respectively. Starting with a silicon $(\mathrm{Si})\langle 100\rangle$ wafer and an appropriate etch mask (either $\mathrm{SiO}_{2}$ or $\mathrm{Si}_{3} \mathrm{~N}_{4}$ ), a perfectly defined $\mathrm{V}$-shaped groove results, with an angle $\alpha=54.73^{\circ}$ (see Fig. 2).

After removal of the mask material the wafer is used as the silicon platform for the optical waveguide definition (see next section). The fabricated channel waveguides are defined parallel to the center of the V-groove (see Fig. 3a). As the depth of the groove is linear with the width of the etch mask, a perfect lateral alignment between channel waveguide and (the core of the) used single-mode fiber can be achieved when the fiber is pressed into the 


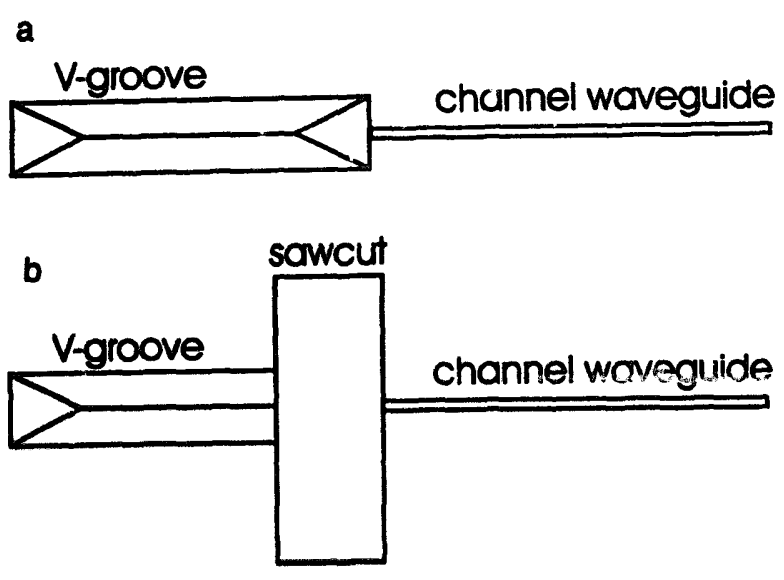

c

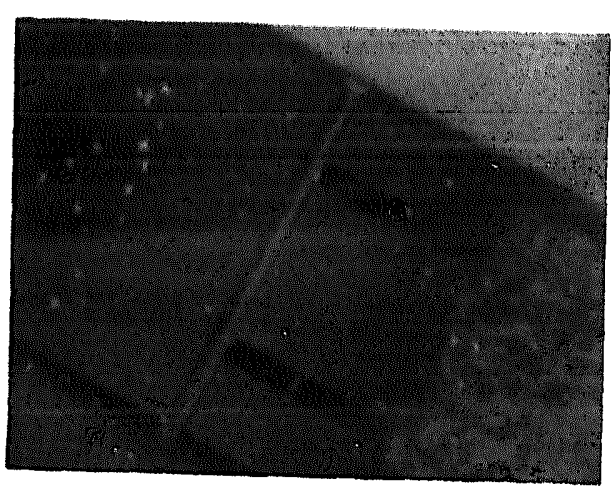

Fig. 3. Schematic representation (top view) of V-groove and channel waveguide before (a) and after (b) the sawing procedure. Photograph of the resulting fiber-to-chip connection unit (c), clearly showing the $V$ groove and the sawcut. A fiber is shown on the left, without yet conlacting the channel waveguide endface.

groove (provided a correction is made for the effect of the deposited waveguide configuration thickness).

After the fabrication of the sensor, the fiber-to-chip connection unit can be completed. For this purpose the Vgroove plane perpendicular to its axis has to be removed: this is done after the deposition of the complete waveguiding layer configuration (see Section 4). This is achieved using a sawing machine (Micro Automation model 1006). As the sawcut width is only approximately $50 \mu \mathrm{m}$ (saw blade width $40 \mu \mathrm{m}$ ), several repetitions have to be made. The depth of the sawcut is typically $100 \mu \mathrm{m}$. The resulting sawcut not only defines the endface of the channel waveguide, but also allows for the necessary close physical contact between fiber and channel (see Fig. 3).

Finally the fiber is fixed into the groove, using a transparent. UV curable glue with refractive index 1.5 (Permacol BV, type 27/3). The glue shows minimal shrinkage during curing.

\section{Sensur design and waveguide configuration}

\subsection{Waveguiding material}

$\mathrm{ZnO}$ was used as the waveguiding material. Its high re- fractive index $(-2.0)$ enables a high surface sensitivity, as has been discussed extensively elsewhere $[12,13]$. Furthermore, $\mathrm{ZnO}$ shows the electro-optic effect: by applying an electric field across a layer of this material, the optical refractive index is changed. Used as waveguiding material, the corresponding phase of the guided mode can be changed using an appropriate electrode configuration: the sign of this phase change depends on the direction of the applied electric field.

\subsection{Wavelength}

The surface sensitivity of sensors using the evanescent field of an optical waveguides $[12,13]$, as well as the typically required electro-optical modulation voltage [6], are inversely linear with the wavelength used. The smallest wavelength possible when using $\mathrm{ZnO}$ is $\approx 600 \mathrm{~nm}$ : below this value the waveguide attenuation sharply increases [15]. Therefore, the (commercially attractive) He-Ne laser with wavelength $\lambda=633 \mathrm{~nm}$ is used as optical source.

\subsection{Mach-Zehnder interferometer geometry}

In Fig. 4a, the layout of the designed MZI is schematically depicted. Although one sensor window and one top electrode would also do, there are two reasons for using the shown crosswise symmetrical design. First, the MachZehnder geometry and therefore the sensor output is now completely balanced. Secondly, for most sensing applications an easily accessible reference branch is desirable (testing weights and measures).

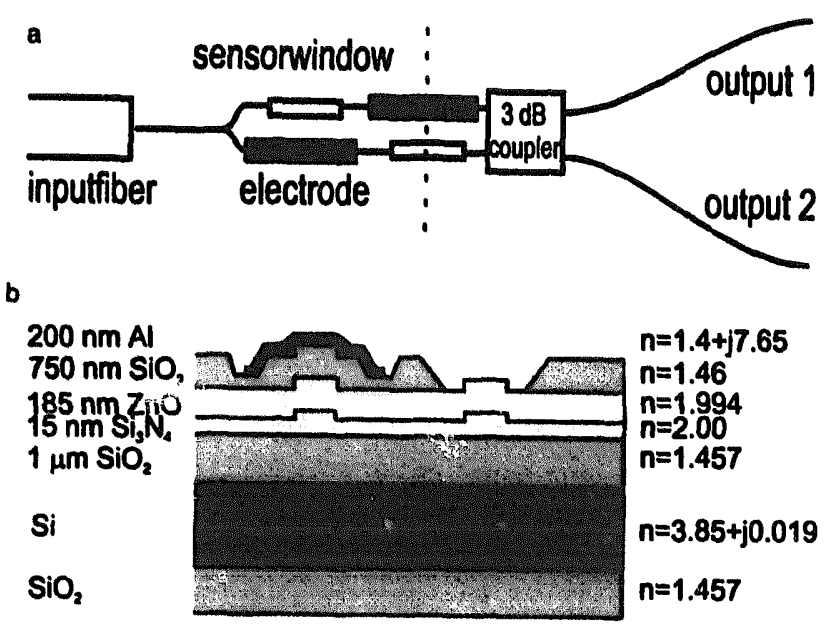

Fig. 4. Schematic representation (top view) of the complete device, showing the input fiber, the MZI and the output channel waveguides (a). For explanation, see text. The cross-section corresponding to the dotted line in the top view representation is shown in (b). The parameters are given for a wavelength of $633 \mathrm{~nm}$. The two top electrodes can be driven individually; the grounded Si-wafer is used as the bottom electrode. The layout is crosswise symmetric to keep the sensor as balanced as possible. One of the sensor windows can be used as a reference. 
The (single-mode) waveguide channel width is $4 \mu \mathrm{m}$, with a minimal applicable channel curve radius of $50 \mathrm{~mm}$ for the TE polarization. The distance between two oppcsite fiber-to-chip connector units is standardized to be $4 \mathrm{~cm}$. The lengths of the electrodes and sensor windows are $9 \mathrm{~mm}$ and $5 \mathrm{~mm}$, respectively.

For linearizing the electro-optically modulated sensor signal it is convenient to obtain two MZI output signals which are mutually out of phase [7]. Therefore as 3-dB coupler (see Fig. 4a) a $2 \times 2$ MMI (multimode interference coupler [14]), is used as 3-dB coupler (see Fig. 4a), resulting in

$I_{\text {output I }} \sim I_{\text {input }}(1+\sin (\Delta \varphi))$

$I_{\text {output } 2} \sim I_{\text {input }}(1-\sin (\Delta \varphi))$

With the waveguide configuration used, the MMI length, designed for TE polarization, is $4287 \mu \mathrm{m}$ and its width is $45 \mu \mathrm{m}$. Its output channels are curved to a final mutual distance of $500 \mu \mathrm{m}$.

\subsection{Waveguide configuration and layer thicknesses}

Thin layers of sputtered $\mathrm{ZnO}$ show a polycrystalline structure with its columnar ( $c$-)axis perpendicular to the substrate while the columns show a random orientation parallel to the substrate [15]. Only an external electric field component perpendicular to the substrate will therefore result in a macroscopic refractive index change [6].

To obtain the desired vertical electric field, both interferometer branches are covered with a top electrode, with the grounded Si-wafer used as bottom electrode. The two top electrodes can be driven individually. The optical phase difference between the two interferometer branches determines the light intensity in each of the output channels (Eq. (1)). The voltage $\times$ length product necessary to switch all the light from output 1 to output 2 (see Eq. (2)) is called $V_{\pi}$.

For accuracy reasons, the waveguide channels - with a ridge height of only $1.5 \mathrm{~nm}$ - are defined in a very thin layer of $\mathrm{Si}_{3} \mathrm{~N}_{4}$, having the same refractive index as $\mathrm{ZnO}$. As a trade-off between sensor sensitivity and electro-optic efficiency, a waveguide layer thickness of $200 \mathrm{~nm}(15 \mathrm{~nm}$ $\mathrm{Si}_{3} \mathrm{~N}_{4}$ and $185 \mathrm{~nm} \mathrm{ZnO}$ ) is used, as a compromise between maximal sensitivity $[4,13]$ and minimal required $V_{\pi}$ voltage [6]. The latter also implies a minimal thickness for both $\mathrm{SiO}_{2}$ layers: with the values given in Fig. $4 \mathrm{~b}$ the combined electrode and silicon buffer induced attenuation is calculated to be less than $0.2 \mathrm{~dB} \mathrm{~cm}^{-1}$. This theoretical increase is regarded as acceptable by the authors compared to the experimentally found $\sim 3 \mathrm{~dB} \mathrm{~cm}^{-1}$ channel attenuation.

To minimize the influence of light not coupled, the channel waveguides (e.g. slabmodes), the $\mathrm{SiO}_{2}$ layer thickness next to the channels is reduced to $250 \mathrm{~nm}$ (see Fig. 4b): the equivalent aluminum induced attenuation of these slab modes now exceeds $20 \mathrm{~dB} \mathrm{~cm}^{-1}$.

\subsection{Sensor window definition}

For the sensor window definition, a two-step etch procedure is used. First, the mentioned (slabmodes suppression step) 'wet' etch of $500 \mathrm{~nm}$ of the top $\mathrm{SiO}_{2}$ layer is used, leading to nicely tapered smooth edges. The remaining $\mathrm{SiO}_{2}$ layer is removed using a reactive ion etch (RIE) procedure which does not attack $\mathrm{ZnO}$. It is important not to etch too deep with the first step, as $\mathrm{ZnO}$ is very reactive and immediately attacked by almost all acids.

\section{Fabrication and packaging}

The fabrication of the optical chips can be divided into two major parts: (a) V-groove fabrication and (b) fabrication of waveguide configuration.

After these two fabrication steps the necessary sawcut to complete the fiber-to-chip connection unit is made, followed by sawing the wafer into the individual optical chips. Then the electrodes are connected and the fiber is glued into the groove. The following processing steps were used.

\subsection{V-Groove fabrication}

(1) Oxidation of silicon $\langle 100\rangle$ wafer at $1150^{\circ} \mathrm{C}$ resulting in $1000 \mathrm{~nm} \mathrm{SiO} 2$.

(2) Photolithograpical definition of V-groove etch mask.

(3) Etching of $\mathrm{SiO}_{2}$ using an $\mathrm{HF} / \mathrm{NH}_{4} \mathrm{~F}$ 1:7 solution (BHF).

(4) Removal of photoresist.

(5) Etching of V-grooves with a $\mathrm{KOH}$-solution: Vgroove width $145 \mu \mathrm{m}$.

(6) Removal of $\mathrm{SiO}_{2}$ with BHF.

\subsection{Waveguide fabrication}

(7) Growing of oxide buffer layer at $1150^{\circ} \mathrm{C}$ resulting in $1000 \mathrm{~nm} \mathrm{SiO} 2$.

(8) Growing of $15 \mathrm{~nm} \mathrm{Si}{ }_{3} \mathrm{~N}_{4}$ using low pressure chemical vapor deposition (LPCVD) at $800^{\circ} \mathrm{C}$.

(9) Photolithographical definition of MZI sensor structure.

(10) Definition of MZI structure (ridge height $1.5 \mathrm{~nm}$ ) by BHF etching.

(11) Removal of photoresist.

(12) Growing of $\mathrm{ZnO}$ layer with thickness of $185 \mathrm{~nm}$ (radio frequency (RF) planar magnetron sputtering $[15]$ at $600^{\circ} \mathrm{C}$ ).

(13) Growing of covering $\mathrm{SiO}_{2}$ layer of $750 \mathrm{~nm}$ using plasma enhanced chemical vapor deposition (PECVD) at $3 \mathrm{O}^{\circ} \mathrm{C}$. 


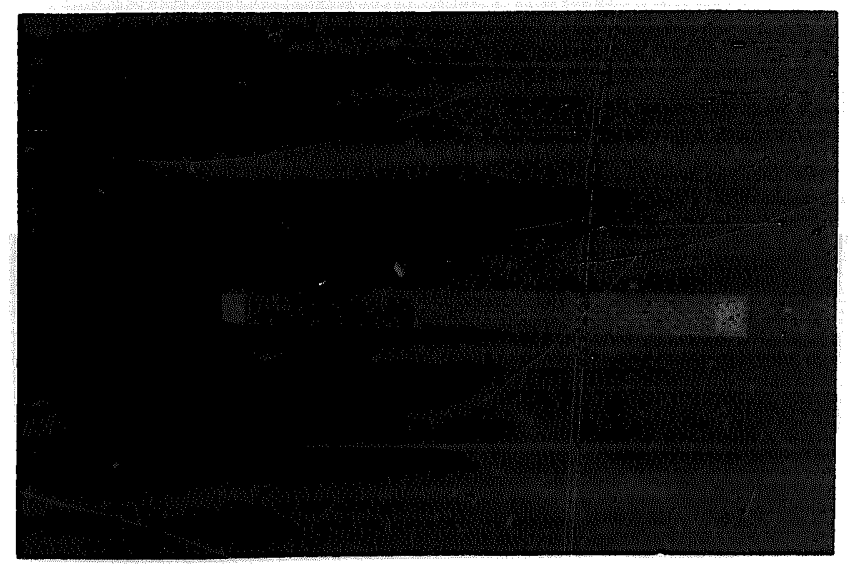

Fig. 5. Photograph of a typical optical chip used for the experiments. At the opposite ends of the chip the V-grooves and the sawcut, forming the fiber-to-chip connection unit, can be seen.

(14) Removal of $500 \mathrm{~nm} \mathrm{SiO}$ using BHF, for slabmode attenuation purposes and sensor window definition.

(15) Definition of the (negative) electrode structure and slabmode attenuators by photolithography.

(16) Growing of $200 \mathrm{~nm}$ aluminum ( $\mathrm{Al}$ ), using electron beam sputtering.

(17) Lift off of the aluminum.

(18)Finishing of the sensor window definition, by removal of the remaining $250 \mathrm{~nm} \mathrm{SiO}{ }_{2}$ using reactive ion etching (RIE).

(19) Completion of the fiber-to chip connection unit by applying the sawcut (see Section 5.1).

(20) Sawing of the optical chips.

A photograph is shown of a completed optical chip in Fig. 5.

This optical chip is now fixed onto a motherboard with copper wiring, after which the electrodes are contacted through wire bonding (apparatus: WeldEquip Westbond). Then the incoupling fiber is glued into the connection unit (see Section 5.1) using home made apparatus. The fiber (manufacturer: Optical Waveguide Systems) is single mode for the $633 \mathrm{~nm}$ wavelength used, having a core diameter of $4 \mu \mathrm{m}$ and (standard) an outer diameter of $125 \mu \mathrm{m}$. For the outcoupling, the same procedure can be applied, using multimode fibers. However, for convenience in characterizing the individual components of the device, the opposing connector unit is removed, and channel outputs are focused onto a detection unit (photodiodes and/or power meter) using a microscope objective (see Fig. 6).

\section{Results and discussion}

\subsection{Fiber-to-chip-connection unit}

For the single-mode fiber used, it can be calculated that the V-groove width should be approximately $145 \mu \mathrm{m}$ for maximal fiber-to-chip coupling efficiency. This was

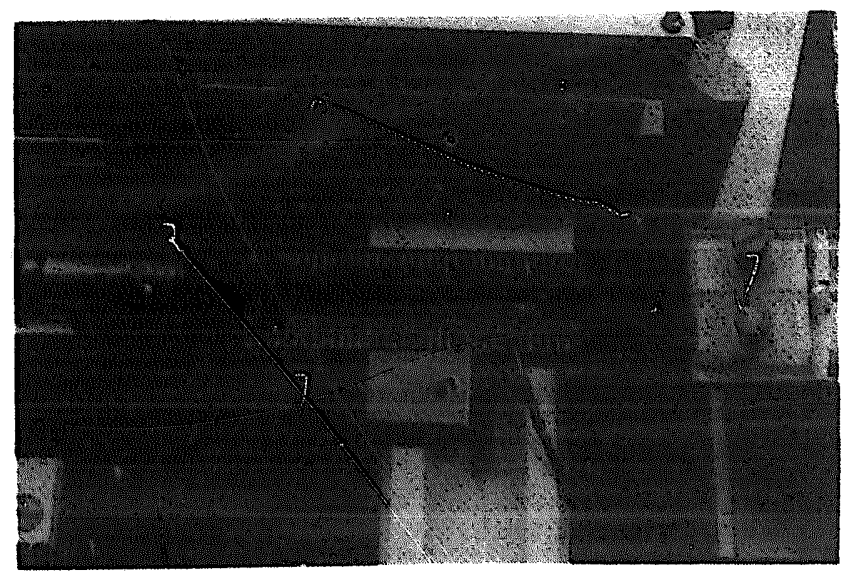

Fig. 6. Photograph of a packaged device, showing the copper wires (wire bonded to the electrodes), and the connected fiber used for the incoupling. The outcoupling fiber-to-chip connection unit is removed; with a microscope objective, both sensor output channels are focused onto a detection usit.

indeed demonstraied: compared with a V-groove width $145 \mu \mathrm{m}, 140 \mu \mathrm{m}$ and $150 \mu \mathrm{m}$ showed strongly reduced coupling efficiency, while at $135 \mu \mathrm{m}$ and $155 \mu \mathrm{m}$ no coupling at all could be realized.

With a V-groove width of $145 \mu \mathrm{m}$, the theoretical coupling efficiency between the used fiber and waveguide configuration can be calculated (computer program 'Couple', manufactured by BBV) to be $\approx 10 \%$. The throughput of the device is measured experimentally. After careful correction for the waveguide attenuation the coupling efficiency can now be calculated to be typically $0.1-1 \%$ (see Table 1). Here the waveguide attenuation is determined using the cut-back method [16] to be typically $3 \mathrm{~dB} \mathrm{~cm}^{-1}$ at $633 \mathrm{~nm}$.

From these values, it can be concluded that the connection unit, although easy to use, results in far from optimal coupling efficiencies. Although the typical experimental coupling efficiency is found to be sufficient for sensor applications, effort will be put in obtaining increased efficiencies through varying the exact $V$-groove width. Furthermore, as the theoretical coupling efficiency is strongly dependent on the waveguide thickness, an adiabatic tapering can result in largely increased coupling efficiencies.

Table 1

Typical fiber-to-chip coupling and electro-optical phase modulation results of a prototype packaged device

\begin{tabular}{|c|c|c|c|}
\hline \multicolumn{2}{|c|}{ Coupling efficiency } & \multicolumn{2}{|c|}{ Electro-optic modulation at $2 \mathrm{kHz}$} \\
\hline $\begin{array}{l}\text { Theoretical } \\
\text { (\%) }\end{array}$ & $\begin{array}{l}\text { Experimental } \\
(\%)\end{array}$ & $\begin{array}{l}V_{\pi} \\
\left(V_{\mathrm{cm}}\right)\end{array}$ & $\begin{array}{l}\text { Modulation depth } \\
\text { (dB) }\end{array}$ \\
\hline 10 & $0.1-1$ & $15 \pm 4$ & $\leq-7$ \\
\hline
\end{tabular}




\subsection{Electro-optical phase modulation}

In the phase modulation experiments the voltage is applied between one of the top electrodes and the bottom electrode. Typically, the maximum intensities measured in both output channels differ less than $5 \%$. The modulation depth, being defined as $10 \times \log \left(I_{\min } / I_{\max }\right) \mathrm{dB}$, is measured, in combination with $V_{\pi}$. The experimental results obtained at an applied frequency of $2 \mathrm{kHz}$ are summarized in Table 1.

When using an inverted modulation voltage on the second top electrode (see Fig. 4a), a voltage of only $8 \mathrm{~V}$ will be needed to obtain complete optical switching between the two output channels! This remarkably small voltage can be even further reduced through optimization of the waveguide structure and electrode structure. The typical modulation depth of $-7 \mathrm{~dB}$ is sufficient for sensor applications.

In Fig. 7 the $V_{\pi}$ voltage is shown as a function of the frequency. From this figure it follows that the electrooptical phase modulation is only applicable from (approximately) $100 \mathrm{~Hz}$, which is due to the electronic conduction of $\mathrm{ZnO}$. The upper frequency boundary of $\approx 10 \mathrm{kHz}$ is due to the $\mathrm{RC}$ time related to the electrodes and can therefore be easily increased. Furthermore, this value is fast enough for most sensing applications.

Using an ac-modulation with amplitude $V_{\pi}$, the sensor output signal can be transformed into a linearized signal using a simple electronic scheme; the sensor output of an ac-modulated sensor can be transformed into a linearized signal with increased sensor sensitivity. It can be estimated that the minimal detectable phase change $\Delta \varphi_{\min }$ can be $1 \times 10^{-3} \times 2 \pi[3,4,7]$, compared to $5 \times 10^{-2} \times 2 \pi$ with a passive interferometer (see next paragraph).

\subsection{General waveguide sensitivity measurements}

In order to verify the intrinsic sensitivity of the optical waveguide, some preliminary experiments were carried out with a completed sensor. Here a refractive index

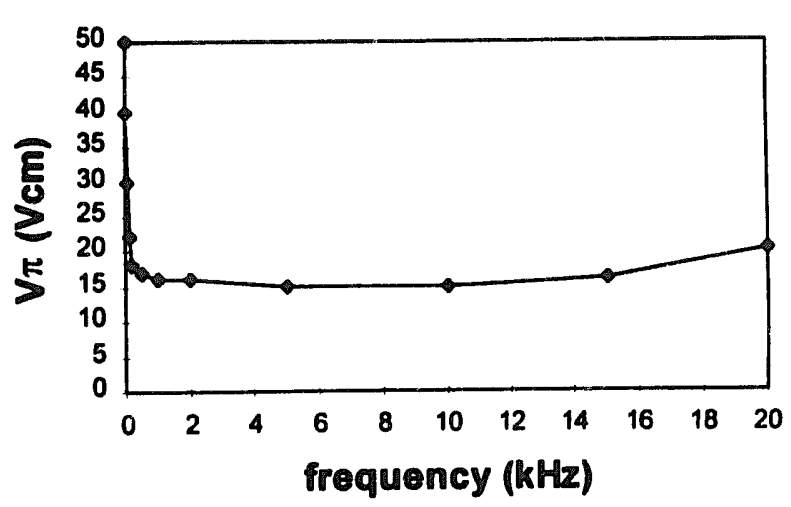

Fig. 7. The measured voltage $\times$ length product $V_{\pi}$ as a function of frequency.

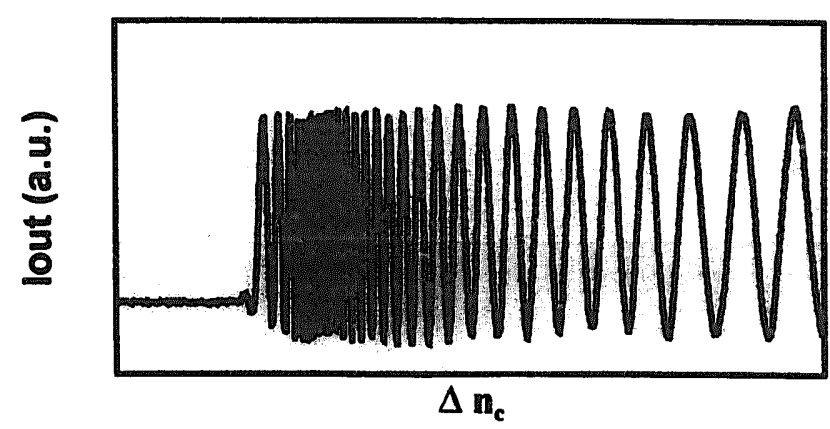

Fig. 8. Measured output intensity of one of the output channels of the passive MZI, as a result of a refractive index change $\Delta n_{\mathrm{c}}$ of $\sim 5 \times 10^{-2}$. The sensor window length is $5 \mathrm{~mm}, \lambda=632.8 \mathrm{~nm}$, and the $\mathrm{ZnO}$ waveguide thickness is $\approx 200 \mathrm{~nm}$.

change in only one of the sensing windows is introduced. No selective transducer layer was applied: furthermore, the Mach-Zehnder interferometer was not electrooptically modulated. The intensity of one of the outputs of the interferometer was monitored as a function of the

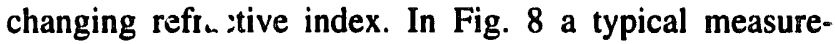
ment is shown.

The measured general sensitivity is found to be in agreement with theory: approximately 1 fringe (phase change of $2 \pi$ ) per $1.5 \times 10^{-3}$ change in the refractive index. This can be calculated to be equivalent to the growth of a protein layer of $1 \mathrm{~nm}$, being a fraction $1 / 5$ of a monolayer of typical antibody proteins $[4,12]$.

For this passive MZI sensor, the minimal detectable phase change $\Delta \Phi_{\min }$ can be estimated to be $5 \times 10^{-2} \times 2 \pi$ [17]. For our waveguide configuration this means a sensitivity of $\approx 7 \times 10^{-5}$ change in bulk refractive index unit, or, in terms of proteins, equivalent to the growth of an (averaged) layer thickness of $0.5 \AA$. Using the sensitivity estimation corresponding to the actively modulated MZI, these values would be $\approx 1 \times 10^{-6}$ and $1 \times 10^{-2} \AA$ (!), respectively.

\section{Conclusions}

In this paper we have described the design and fabrication of a packaged chemo-optical sensor based on $\mathrm{ZnO}$ optical waveguides. The sensor consists of a sensitive waveguide Mach-Zehnder interferometer. Preliminary refractive index measurements show the high general sensitivity of the passive sensor: a phase shift of $2 \pi$ per $1.5 \times 10^{-3}$ change in the refractive index. The corresponding sensitivity of such a passive interferometer, with the minimal detectable phase shift $\Delta \varphi_{\min }$ estimated as $5 \times 10^{-}$ $2 \times 2 \pi$ [17], can be calculated to be equivalent to the growth of $5 \times 10^{-2} \mathrm{~nm}$ of a typical layer of antibody proteins $[4,12]$ with the applied waveguide corifiguration.

The use of $\mathrm{ZnO}$ as optical waveguide enables electrooptical modulation of the sensor signal. The measured modulation voltage $\times$ length product $V_{\pi}$ is $15 \pm 4 \mathrm{~V} \mathrm{~cm}$ 
for the TE-polarized light used in the frequency range between $100 \mathrm{~Hz}$ and $10 \mathrm{kHz}$. With the Mach-Zehnder interferometer configuration used, a driving voltage of only $\approx 8 \mathrm{~V}$ is necessary to obtain complete optical switching between the two output signals. The upper frequency limit - being high enough for most sensor applications - is caused by the RC time related to the electronics and can therefore be easily increased. The lower limit of $100 \mathrm{~Hz}$ is due to electronic conduction in the $\mathrm{ZnO}$ layer. The modulation depth is typically $\leq-7 \mathrm{~dB}$.

With a simple electronic scheme, the sensor output of such an actively modulated sensor can be transformed into a linearized signal, with increased sensor sensitivity. The corresponding minimal detectable phase change, estimated as $1 \times 10^{-3} \times 2 \pi[3,4,7]$, is equivalent to $1 \times$ $10^{-3} \mathrm{~nm}$ of antibody proteins.

The sensor is supplied with an easy-to-use fiber-tochip connection unit. This unit is essentially based on silicon V-grooves combined with a smooth sawcut, defining the endface of the channel waveguides used. The measured coupling efficiency between the fiber used and the sensor chip, $0.1-1 \%$, is found to be considerably lower than the theoretical value $(\approx 10 \%)$. As a high coupling efficiency is not necessary for most sensing applications, this is regarded as sufficient by the authors. Nevertheless, effort will be put into increasing this value.

In a next stage, the sensor will be activated, using the electro-optic modulation shown. Combined with an appropriate electronic scheme, the sensor signal will be linearized. The corresponding accurate measurements will be carriad out with this sensor, involving selective transducer layers.

The reported device, although by no means fully optimized, is a very sensitive prototype chemo-optical sensor. In a next stage, selective transducer layers will be incorporated, together with the improved, actively modulated sensor readout.

\section{Acknowledgements}

These investigations were supported by the Dutch Innovative Research Program (IOP) Electro-Optics. The authors want to thank Ing. T. Andringa for the fabrication of the optical chips.

\section{References}

[1] P.V. Lambeck, Integrated opto-chemical sensors, Sensors and Actuators B, 8 (1991) 103-116.

[2] O. Parriaux, in O.S. Wolfbeiss (ed.), Fiberoptic Chemical Sensors and Biosensors, Ch. 4., CRC Press, Boca Raton, FL, 1991.

[3] W. Lukosz, Principles and sensitivities of integrated optical and surface plasmon sensors for direct affinity sensing and immunosensing, Biosensors and Bioelectronics, 6 (1991) 215-225.

[4] R.G. Heideman, R.P.H. Kooyman and J. Greve, Performance of a highly sensitive optical waveguide Mach-Zehnder interferometer immunosensor, Sensors and Actuators B, 10 (1993) 209-217.

[5] J. van Gent, Surface Plasmon resonance-based chemo-optical sensors, Ph.D. Thesis, University of Twente, 1990.

[6] R.G. Heideman, R. Lammers and P.V. Lambeck, Electro-optical phase modulation in zinc oxide $(\mathrm{ZnO})$, Proc. National Senser Conference, Delft, The Netherlands, 1996, pp. 231-234.

[7] P.V. Lambeck, R.G. Heideman and T.J. Ikkink, Phase-modulated Mach-Zehnder interferometer for sensor applications, Proc. IOth Nordic Baltic Conf., Finland, 1996.

[8] O.G. Ramer, Single-mode fiber-to-channel waveguide coupling, J. Opt. Commun., 2 (1981) 122-127.

[9] G.E. Henein, J.V. Gates, L.J. Mulligan, H.M. Presby and J.F. de Jong, Fiber to laser coupling on silicon optical bench platform, Proc. Integrated Photonics Research. Boston. MA. 1996. pp. 396-399.

[10] R. Moosburger, B. Schüppert, U. Fisher and K. Petermann, Passive alignment technique for all-silicon integrated optics, Proc. Integrated Photonics Research, Boston, MA, 1996, pp. 565-568.

[11] O.J. Glembochi and R.E. Stahlbush, Bias-dependent etching of silicon in aqueous KOH, J. Electrochem. Soc., 132 (1985) 145158.

[12] R.G. Heideman, R.P.H. Konyman and J. Greve, Immunoreactivity of adsorbed anti human chorionic gonadotropin ( $\alpha \mathrm{hCG}$ ) studied with an optical waveguide interferometric sensor, Biosensor Biselectron., 9 (1994) 33-43.

[13] K. Tiefentahler and W. Lukosz, Sensitivity of grating couplers as integrated-optical chemical sensors, J. Opt. Soc. Am. B., 6 (1989) 209-220.

[14] L.B. Soldano and E.C.M. Pennings, Optical multi-mode interference devices based on self-imaging: principles and applications, J. Lighnwave Technol., 13 (1995) 615-627

[15] R.G. Heideman, P.V. Lambeck and J.G.E. Gardeniers, High quality $\mathrm{ZnO}$ layers with adjustable refractive indices for integrated optics applications, Opt. Mater., 4 (1995) 741-755.

[16] G.T. Reed, Methods of measurement of passive integrated optical waveguides, Proc. IEE Colloquium on Measurements on Optical Devices, Digest No. 210, London, UK, 1992, pp. 2/1-7.

[17] R.G. Heideman, R.P.H. Kooyman and J. Greve, A simple interferometer for evanescent field refractive index sensing as a feasibility study for an immunosensor. Appl. Opt., 30 (1991) 1474 1479. 